\title{
PROHIBITING ISOLATED SINGULARITIES IN OPTIMAL TRANSPORT
}

\author{
YOUNG-HEON KIM AND JUN KITAGAWA
}

\begin{abstract}
We give natural topological conditions on the support of the target measure under which solutions to the optimal transport problem with cost function satisfying the (weak) Ma, Trudinger, and Wang condition cannot have any isolated singular points.
\end{abstract}

\section{MAIN RESUlts}

The optimal transport problem is the following: given source and target probability measure spaces $(\Omega, \mu),(\bar{\Omega}, \nu)$, and a measurable cost function $c: \Omega \times \bar{\Omega} \rightarrow \mathbb{R}$, find an optimal measurable mapping $T: \Omega \rightarrow \bar{\Omega}$ defined $\mu$-a.e., minimizing

$$
\int_{\Omega} c(x, F(x)) \mu(d x)
$$

over the set of all measurable $F: \Omega \rightarrow \bar{\Omega}$ with $F_{\#} \mu=\nu$.

A fundamental problem in optimal transport theory is to understand the regularity of optimal maps. In the classical case where the cost function is given by the quadratic $\operatorname{cost} c(x, \bar{x})=|x-\bar{x}|^{2} / 2$ on $\mathbb{R}^{n} \times \mathbb{R}^{n}$ (or equivalently $c(x, \bar{x})=-\langle x, \bar{x}\rangle$ ), it is well known that the optimal map is Hölder continuous [5] 7 if the support of the target measure is convex, for source and target measures with densities bounded above and below. For more general cost functions one would require certain structural conditions, namely, (Twist), (Nondeg and especially, the $c$-convexity of the support of the target measure and the condition (MTW) 24,27] which are shown to be necessary 21,24 for regularity theory of the classical case to be extended (see Section 2 for relevant definitions). Under these conditions, Hölder continuity of the optimal map is known, under the assumption that the source and target measures have densities bounded from above and below, see [12, 14, 19, 21. For smoother measures, higher regularity theory is also known, see [20,24, 27.

A natural question one can ask is what happens if one of the above structural conditions is violated. In particular, we focus on the geometric condition of convexity / $c$-convexity of the support of the target measure, where it is known that without such conditions optimal maps may not be continuous [7,24].

2010 Mathematics Subject Classification. 35J96.

Y.-H.Kim's research is partially supported by Natural Sciences and Engineering Research Council of Canada Discovery Grants 371642-09 as well as the Alfred P. Sloan Research Fellowship 2012-2014. Both of the authors were also supported by the Mathematical Sciences Research Institute (MSRI) as members in the thematic program "Optimal Transport: Geometry and Dynamics". Part of this work was done while Y.-H.Kim was visiting University Paris-Est Créteil (UPEC) and Korea Advanced Institute of Science and Technology (KAIST).

(C) 2014 by the authors. 
As a first step in this direction, in this paper we analyze the case of isolated singular points (thoughout this paper, by singularity or singular point we indicate a point where a function is not differentiable). In the following main theorem we prove that if the support of the target measure has no holes (by which we mean a bounded, open, connected component of the complement of the target domain), then the corresponding Brenier solution cannot have an isolated singular point in the interior of the support of the source measure. The precise statement follows with the relevant definitions given in Section 2 .

Theorem 1.1. Let $M$ and $\bar{M}$ be $n$-dimensional Riemannian manifolds and $\Omega$ and $\bar{\Omega}$ be bounded domains in $M$ and $\bar{M}$, respectively. Let $c$ be a cost function $c: \Omega \times \bar{\Omega} \rightarrow \mathbb{R}$ that satisfies (A0), (Twist), (Nondeg), and (MTW), and assume that $\Omega$ and $\bar{\Omega}$ are c-convex with respect to each other.

Consider two absolutely continuous probability measures $\mu=f \mathrm{dVol}$ and $\nu=$ $g \mathrm{dVol}$ on $M$ and $\bar{M}$, respectively, with supports spt $\mu \subset \Omega$ and spt $\nu \subset \bar{\Omega}$. Assume that $\operatorname{spt} \nu \cap \bar{\Omega}^{\partial}=\emptyset$ and that there exists a constant $0<\Lambda<\infty$ such that

$$
\Lambda^{-1} \leq f, g \leq \Lambda
$$

on their supports.

Finally, let $u$ be a Brenier solution (see Definition [2.6) to the optimal transport problem with cost $c$. For each $x_{0} \in(\operatorname{spt} \mu)^{\mathrm{int}}$, if there are no holes (see Definition 2.11) in spt $\nu$ that are c-convex with respect to $x_{0}$, then $x_{0}$ cannot be an isolated singular point of $u$.

Remark 1.2. Examples of cost functions satisfying (A0), (Twist), (Nondeg), and (MTW), can be found in [18, 22, 24, 27, see also [28].

In the special case when $M$ and $\bar{M}$ are domains in Euclidean space and $c(x, \bar{x})=$ $\frac{1}{2}|x-\bar{x}|^{2}, c$-convexity reduces to ordinary convexity (we will henceforth refer to this setting as the Euclidean case). In the two dimensional Euclidean case, Figalli [9] studied the geometric structure of the singular set, and the above result on isolated singularity follows as a special case. In higher dimensions, it seems that no result on the geometric structure of singular sets (similar to the one in [9]) is currently known. For some previous related works in the Euclidean case, see [2, 16], [29], and [8, Section 5] in the case of dimension 2, and [3, 25] in higher dimensions.

While the other results mentioned above consider isolated singularities of the Monge-Ampère equation, the papers [29] and 8, Section 5] deal specifically with the case of the optimal transport problem (however, still in the Euclidean case). Both results discuss the finer question of Lipschitz or $C^{1}$ propagation of singularities, but assume stronger conditions in addition to topological restrictions on the support of the target measure. Specifically, 29] assumes that all singular points have a subdifferential of affine dimension at most one, while [8] requires the support of the source measure be convex. Our main result applies to a more general class of $c$, and also requires no hypothesis on spt $\mu$; in fact we obtain the condition required for [29] in the course of our proof (see Proposition [3.2).

Throughout this paper, we will denote the closure, interior, and boundary of a set $A$ by $A^{\mathrm{cl}}, A^{\mathrm{int}}$, and $A^{\partial}$ respectively. 


\section{RELEVANT DEFINITIONS AND PRELIMINARIES}

In this section we gather some relevant definitions and facts about $c$-convex potential functions in relation to solutions of the optimal transport problem. Some good references are [15,28].

Let $M$ and $\bar{M}$ be $n$-dimensional Riemannian manifolds and $\Omega$ and $\bar{\Omega}$ be bounded domains in $M$ and $\bar{M}$, respectively. Let $c$ be a measurable cost function $c: \Omega \times \bar{\Omega} \rightarrow$ $\mathbb{R}$. We start out by stating the various assumptions we may require on our cost function $c$.

\section{Smoothness of cost:}

$$
c \in C^{4}\left(\Omega^{\mathrm{cl}} \times \bar{\Omega}^{\mathrm{cl}}\right) .
$$

\section{Twist:}

We will say $c$ satisfies condition (Twist) if each of the mappings

$$
\begin{array}{ll}
\bar{\Omega} \ni \bar{x} & \mapsto-D c\left(x_{0}, \bar{x}\right) \in T_{x_{0}}^{*} M, \\
\Omega \ni x & \mapsto-\bar{D} c\left(x, \bar{x}_{0}\right) \in T_{\bar{x}_{0}}^{*} \bar{M},
\end{array}
$$

are injective for each $x_{0} \in \Omega$ and $\bar{x}_{0} \in \bar{\Omega}$. Here, $D, \bar{D}$ denote the usual differential in the $x$ or $\bar{x}$ variable.

Remark 2.1. We use the standard notation $\exp _{x_{0}}^{c}(\cdot)$ and $\exp _{\bar{x}_{0}}^{c}(\cdot)$ to denote the inverses of the above two mappings. Also, for any $A \subset \Omega, \bar{x} \in \bar{\Omega}$ or $\bar{A} \subset \bar{\Omega}, x \in \Omega$, we will write

$$
\begin{aligned}
{[A]_{\bar{x}}: } & =-\bar{D} c(A, \bar{x}), \\
{[\bar{A}]_{x}: } & =-D c(x, \bar{A}) .
\end{aligned}
$$

We also comment here, for the cost $c(x, \bar{x})=-\langle x, \bar{x}\rangle$ on $\mathbb{R}^{n} \times \mathbb{R}^{n}$, these mappings are both just the identity map.

Definition 2.2 (c-convexity of a set [24]). If $A \subset \Omega$ and $\bar{x} \in \bar{\Omega}$, we say that $A$ is c-convex with respect to $\bar{x}$ if the set $[A]_{\bar{x}}$ is a convex subset of $T_{\bar{x}}^{*} \bar{M}$. If $\bar{A} \subset \bar{\Omega}$ and $x \in \Omega$, we define when $\bar{A}$ is c-convex with respect to $x$ and $A$ and $\bar{A}$ are $c$-convex with respect to each other in the obvious way.

\section{Nondegeneracy:}

We say $c$ satisfies condition (Nondeg) if, for each $x \in \Omega$ and $\bar{x} \in \bar{\Omega}$, the linear mapping

$$
-\bar{D} D c(x, \bar{x}): T_{\bar{x}} \bar{M} \rightarrow T_{-D c(x, \bar{x})}\left(T_{x}^{*} M\right) \cong T_{x}^{*} M \quad \text { (Nondeg) }
$$

is invertible (and consequently, so is its adjoint mapping, $-D \bar{D} c(x, \bar{x}): T_{x} M \rightarrow$ $\left.T_{\bar{x}}^{*} \bar{M}\right)$.

MTW (Ma-Trudinger-Wang condition) 21,24,27]: We say $c$ satisfies the condition (MTW if, for any $x \in \Omega, \bar{x} \in \bar{\Omega}$, and $V \in T_{x} M, \eta \in T_{x}^{*} M$ with $\eta(V)=0$,

$$
-\left(c_{i j, p q}-c_{i j, r} c^{r, s} c_{s, p q}\right) c^{p, k} c^{q, l}(x, \bar{x}) V^{i} V^{j} \eta_{k} \eta_{l} \geq 0 .
$$

Here we fix coordinate systems on $M$ and $\bar{M}$ and take all derivatives with respect to these coordinates; lower indices before a comma denote derivatives of $c$ with respect to the $x$ variable, and lower indices after a comma denote derivatives with respect to the $\bar{x}$ variable. Also, a pair of raised indices denotes the inverse of a matrix.

We next define some basic concepts of use in $c$-convex geometry. 
Definition 2.3. A real valued function $u$ defined on $\Omega$ is said to be $c$-convex if for any $x_{0} \in \Omega$, there exists some $\bar{x}_{0} \in \bar{\Omega}$ and $\lambda_{0} \in \mathbb{R}$ such that

$$
\begin{gathered}
-c\left(x_{0}, \bar{x}_{0}\right)+\lambda_{0}=u\left(x_{0}\right), \\
-c\left(x, \bar{x}_{0}\right)+\lambda_{0} \leq u(x)
\end{gathered}
$$

for all $x \in \Omega$. Any function of the form $-c\left(\cdot, \bar{x}_{0}\right)+\lambda_{0}$ is called a $c$-affine function (with focus $\bar{x}_{0}$ ), and if it satisfies the above relations is said to support $u$ from below at $x_{0}$.

We also define the $c$-subdifferential of a $c$-convex function, and the subdifferential of a semi-convex function.

Definition 2.4. The subdifferential of a semi-convex function $u$ at a point $x \in$ $(\operatorname{dom}(u))^{\text {int }}$ is defined by the set

$$
\partial u(x):=\left\{\bar{p} \in T_{x}^{*} M \mid u(x)+\langle v, \bar{p}\rangle+o(|v|) \leq u\left(\exp _{x}(v)\right), T_{x} M \ni v \rightarrow 0\right\},
$$

here $\exp _{x}$ is the Riemannian exponential mapping on $M$.

Similarly, the $c$-subdifferential of a $c$-convex function $u$ at a point $x \in(\operatorname{dom}(u))^{\mathrm{int}}$ is defined as the set

$$
\partial_{c} u(x):=\{\bar{x} \in \bar{\Omega} \mid-c(y, \bar{x})+c(x, \bar{x})+u(x) \leq u(y), \forall y \in \operatorname{dom}(u)\} .
$$

If $A \subseteq \Omega$, we write

$$
\partial_{c} u(A):=\bigcup_{x \in A} \partial_{c} u(x) .
$$

Remark 2.5. Note that if $u$ is semi-convex, each $\partial u(x)$ is a nonempty, convex set, and for any point $x$ where $u$ is differentiable, we have $\partial u(x)=\{D u(x)\}$. Additionally, it is known that if $c$ satisfies (A0), then a $c$-convex function is semiconvex, hence in particular it is differentiable a.e.

Additionally, if $u$ is $c$-convex it is not difficult to see that its $c$-subdifferential is $c$-monotone, i.e. for any $x_{0}, x_{1} \in \Omega$ and $\bar{x}_{0} \in \partial_{c} u\left(x_{0}\right), \bar{x}_{1} \in \partial_{c} u\left(x_{1}\right)$, we have

$$
c\left(x_{0}, \bar{x}_{0}\right)+c\left(x_{1}, \bar{x}_{1}\right) \leq c\left(x_{0}, \bar{x}_{1}\right)+c\left(x_{1}, \bar{x}_{0}\right) .
$$

Definition 2.6. Suppose $c$ satisfies (Twist). A Brenier solution (to the optimal transport problem with cost $c(x, \bar{x}))$ pushing $\mu$ forward to $\nu$ is a $c$-convex function $u$ defined on $\operatorname{spt} \mu$ such that

$$
\begin{aligned}
T_{\#} \mu & =\nu, \\
T(\operatorname{spt} \mu) & \subseteq \operatorname{spt} \nu,
\end{aligned}
$$

where $T$ is the Brenier map defined for a.e. $x$ (where $u$ is differentiable) by

$$
T(x):=\exp _{x}^{c}(D u(x)) .
$$

If $u$ is a Brenier solution pushing $\mu$ forward to $\nu$, then it is well known that $T$ as defined above is optimal in (11).

The following result (discovered by Loeper 21] for domains in $\mathbb{R}^{n}$, further developed in [13, 17, 23, 26, and extended to domains in manifolds under certain conditions) details certain geometric properties of $c$-convex functions. It will play a key role in our main proof. 
Theorem 2.7 (Loeper's maximum principle 21]). Suppose $c, \Omega$, and $\bar{\Omega}$ satisfy the conditions of Theorem 1.1 . Also let $x_{0} \in \Omega, \bar{p}_{0}, \bar{p}_{1} \in[\bar{\Omega}]_{x_{0}}$, and $\bar{x}(t):=$ $\exp _{x_{0}}^{c}\left((1-t) \bar{p}_{0}+t \bar{p}_{1}\right)$. Then for any $x \in \Omega$,

$$
\begin{aligned}
& -c(x, \bar{x}(t))+c\left(x_{0}, \bar{x}(t)\right) \\
& \quad \leq \max \left\{-c(x, \bar{x}(0))+c\left(x_{0}, \bar{x}(0)\right),-c(x, \bar{x}(1))+c\left(x_{0}, \bar{x}(1)\right)\right\} .
\end{aligned}
$$

An analogous inequality holds with the roles of $\Omega$ and $\bar{\Omega}$ reversed.

This lemma has several important consequences, we will require the following two of them later; the second of which was first observed and used in 11, 12 and 19.

Corollary 2.8 ( [21, Theorem 3.1]). Suppose $c$, $\Omega$, and $\bar{\Omega}$ satisfy the same conditions as Theorem 2.7 above, and $u$ is a c-convex function on $\Omega$. Then for any $x_{0} \in \Omega$,

$$
\left[\partial_{c} u\left(x_{0}\right)\right]_{x_{0}}=\partial u\left(x_{0}\right),
$$

in particular, $\partial_{c} u\left(x_{0}\right)$ is c-convex with respect to $x_{0}$.

Corollary 2.9. Suppose $c, \Omega$, and $\bar{\Omega}$ satisfy the same conditions as Theorem 2.7. and $u$ is a c-convex function on $\Omega$. Then, for any $\bar{x}_{0} \in \bar{\Omega}$ and $\lambda_{0} \in \mathbb{R}$, the section

$$
\left\{x \in \Omega \mid u(x) \leq-c\left(x, \bar{x}_{0}\right)+\lambda_{0}\right\}
$$

is c-convex with respect to $\bar{x}_{0}$.

We also state here a fairly standard result concerning $c$-subdifferentials of $c$ cones.

Lemma 2.10. Suppose $c, \Omega$, and $\bar{\Omega}$ satisfy the conditions of Theorem 1.1, $u$ is a c-convex function, $m_{0}$ is a c-affine function with focus $\bar{x}_{0}$, and let $S_{0}:=\left\{u \leq m_{0}\right\}$ be such that $S_{0} \cap \Omega^{\partial}=\emptyset$. Fix $x_{0} \in S_{0}^{\text {int }}$ and define the $c$-cone over the section $S_{0}$ with vertex $x_{0}$ by

$$
K_{x_{0}, S_{0}}^{c}(x):=\sup _{m} m(x),
$$

where the supremum is taken over all c-affine functions $m$ satisfying $m \leq m_{0}$ on $S_{0}^{\partial}$, and $m\left(x_{0}\right) \leq u\left(x_{0}\right)$. Then,

$$
\partial_{c} K_{x_{0}, S_{0}}^{c}\left(x_{0}\right) \subset \partial_{c} u\left(S_{0}\right),
$$

and if $\bar{x}_{0} \in \bar{\Omega}^{\text {int }}$,

$$
-D c\left(x_{0}, \bar{x}_{0}\right) \in\left[\partial_{c} K_{x_{0}, S_{0}}^{c}\left(x_{0}\right)\right]_{x_{0}}^{\text {int }} .
$$

Proof. A proof of (5) is contained in, for example, [14, Lemma 3.4].

We will show (6). By assumption, $m_{0}\left(x_{0}\right)-u\left(x_{0}\right)>0$. Let us write $\bar{p}_{0}:=$ $-D c\left(x_{0}, \bar{x}_{0}\right)$, then recall that $\exp _{x_{0}}^{c}\left(\bar{p}_{0}\right)=\bar{x}_{0}$. Hence for a sufficiently small $r_{0}>0$, we have (for some $C>0$ depending only on derivatives of the $\operatorname{cost} c$ ) that for all $\bar{p} \in B_{r_{0}}\left(\bar{p}_{0}\right)$, the function $m_{\bar{p}}(x):=-c\left(x, \exp _{x_{0}}^{c}(\bar{p})\right)+c\left(x_{0}, \exp _{x_{0}}^{c}(\bar{p})\right)+u\left(x_{0}\right)$ satisfies

$$
\begin{aligned}
m_{\bar{p}}(x) & =\left(-c\left(x, \exp _{x_{0}}^{c}(\bar{p})\right)+c\left(x_{0}, \exp _{x_{0}}^{c}(\bar{p})\right)+m_{0}\left(x_{0}\right)\right)-m_{0}\left(x_{0}\right)+u\left(x_{0}\right) \\
& \leq m_{0}(x)+C r_{0}-\left(m_{0}\left(x_{0}\right)-u\left(x_{0}\right)\right) \\
& <m_{0}(x)
\end{aligned}
$$


for all $x \in S_{0}^{\partial}$. Thus $m_{\bar{p}}$ is admissible in the supremum defining $K_{x_{0}, S_{0}}^{c}$, and it must support the $c$-cone $K_{x_{0}, S_{0}}^{c}$ from below at $x_{0}$. In particular we have for all $\bar{p} \in$ $B_{r_{0}}\left(\bar{p}_{0}\right)$ that $\exp _{x_{0}}^{c}(\bar{p}) \in \partial_{c} K_{x_{0}, S_{0}}^{c}\left(x_{0}\right)$, hence by Corollary 2.8, $\bar{p} \in\left[\partial_{c} K_{x_{0}, S_{0}}^{c}\left(x_{0}\right)\right]_{x_{0}}$, proving (6).

Finally, we give the precise definition of a hole.

Definition 2.11. Given any domain $A$, we say that $\mathcal{O}$ is a hole in $A$ if $\mathcal{O} \neq \emptyset$ is a bounded, open, connected set such that

$$
\begin{aligned}
\mathcal{O} \cap A^{\text {int }} & =\emptyset, \\
\mathcal{O}^{\partial} & \subset A^{\partial} .
\end{aligned}
$$

\section{Proof of Theorem 1.1}

We begin by deriving several intermediate results. We start with stating a very useful tool in our analysis, due to Albano and Cannarsa:

Proposition 3.1 ( [1, Theorem 4.2]). Suppose that $u$ is a semi-convex function and $x_{0} \in(\operatorname{dom}(u))^{\mathrm{int}}$ is a point where $u$ is not differentiable. If there exists an open neighborhood $\mathcal{N}$ of $x_{0}$ such that $u$ is differentiable on $\mathcal{N} \backslash\left\{x_{0}\right\}$, then for every $p \in \partial u\left(x_{0}\right)^{\partial}$ there exists a sequence $x_{k} \rightarrow x_{0}$ such that $D u\left(x_{k}\right) \rightarrow p$ as $k \rightarrow \infty$.

The next result excludes having a full dimensional subdifferential at an isolated singular point, when the target domain contains no holes. Note that the result can be shown under just the condition (Twist), and can be strengthened under (Nondeg) and (MTW. We also comment that this will be the only place where we use the no-hole condition on $\operatorname{spt} \nu$, for the proof of Theorem 1.1.

Proposition 3.2. Suppose that $c$ is $C^{1}$ and satisfies (Twist), $u$ is a c-convex Brenier solution, and $\operatorname{spt} \nu$ contains no holes. Then $u$ cannot have any isolated singular point $x_{0} \in(\operatorname{spt} \mu)^{\mathrm{int}}$ with $\operatorname{affdim} \partial u\left(x_{0}\right)=n$ (here affdim is the affine dimension of a convex set).

If in addition, $c$ satisfies (A0), (Nondeg), and (MTW), and $\Omega$ and $\bar{\Omega}$ are $c$ convex with respect to each other, we obtain the same conclusion under the weaker condition that $\operatorname{spt} \nu$ contains no holes c-convex with respect to $x_{0}$.

Proof. Suppose by contradiction that $x_{0} \in(\operatorname{spt} \mu)^{\mathrm{int}}$ is an isolated singular point of $u$, and the affine dimension of $\partial u\left(x_{0}\right)$ is $n$. Since $c$ is $C^{1}$ and satisfies (Twist), the mapping $\exp _{x_{0}}^{c}(\cdot)$ is continuous and injective, thus Brouwer's invariance of domain theorem (see [4]) gives that $\exp _{x_{0}}^{c}(\cdot)$ is a homeomorphism between the open set $\partial u\left(x_{0}\right)^{\text {int }}$ and its image. In particular, $\exp _{x_{0}}^{c}\left(\partial u\left(x_{0}\right)^{\mathrm{int}}\right)$ is a nonempty, open, bounded, connected set. Then since $x_{0}$ is an isolated singularity, by Proposition 3.1 we have

$$
\exp _{x_{0}}^{c}\left(\partial u\left(x_{0}\right)^{\partial}\right) \subset \operatorname{spt} \nu \cap \partial_{c} u\left(x_{0}\right),
$$

as $D u(\operatorname{dom}(D u)) \subset \operatorname{spt} \nu$ for the Brenier solution $u$.

We now claim that

$$
\exp _{x_{0}}^{c}\left(\partial u\left(x_{0}\right)^{\mathrm{int}}\right) \cap \partial_{c} u\left(x_{1}\right)=\emptyset
$$

for any $x_{1} \neq x_{0}$. First, fix such an $x_{1} \in \Omega$ and define

$$
F(\bar{x}):=c\left(x_{0}, \bar{x}\right)-c\left(x_{1}, \bar{x}\right)
$$


which is a $C^{1}$ function satisfying $D F(\bar{x}) \neq 0$ for any $\bar{x}$ (by (Twist)). In particular, $F$ cannot attain its maximum over the compact set $\exp _{x_{0}}^{c}\left(\partial u\left(x_{0}\right)\right)$ except at the boundary, say at $\bar{x}_{0} \in \exp _{x_{0}}^{c}\left(\partial u\left(x_{0}\right)^{\partial}\right) \subset \partial_{c} u\left(x_{0}\right)$. Thus if there exists $\bar{x}_{1} \in \exp _{x_{0}}^{c}\left(\partial u\left(x_{0}\right)^{\mathrm{int}}\right) \cap \partial_{c} u\left(x_{1}\right)$, this would imply that

$$
\begin{aligned}
F\left(\bar{x}_{1}\right) & <F\left(\bar{x}_{0}\right) \\
\Longleftrightarrow c\left(x_{0}, \bar{x}_{1}\right)-c\left(x_{1}, \bar{x}_{1}\right) & <c\left(x_{0}, \bar{x}_{0}\right)-c\left(x_{1}, \bar{x}_{0}\right) \\
\Longleftrightarrow c\left(x_{0}, \bar{x}_{1}\right)+c\left(x_{1}, \bar{x}_{0}\right) & <c\left(x_{0}, \bar{x}_{0}\right)+c\left(x_{1}, \bar{x}_{1}\right),
\end{aligned}
$$

which is a violation of $c$-monotonicity of the $c$-subdifferential of $u$ (see Remark 2.5). As a result there cannot be such an $\bar{x}_{1}$, and we obtain (8). Since $\nu=T_{\#} \mu$, we must then have

$$
\exp _{x_{0}}^{c}\left(\partial u\left(x_{0}\right)^{\mathrm{int}}\right) \cap \operatorname{spt} \nu=\emptyset .
$$

However, when combined with (17) this exactly implies that $\exp _{x_{0}}^{c}\left(\partial u\left(x_{0}\right)^{\text {int }}\right)$ is a hole in spt $\nu$ which contradicts our initial assumption, therefore it must be that $\operatorname{affdim} \partial u\left(x_{0}\right)<n$.

If $c$ also satisfies (A0), (Nondeg, and (MTW), by Corollary 2.8 we have that $\exp _{x_{0}}^{c}\left(\partial u\left(x_{0}\right)^{\text {int }}\right)=\partial_{c} u\left(x_{0}\right)^{\text {int }}$ and is $c$-convex with respect to $x_{0}$; the conclusion thus follows from the same proof as above.

In the next lemma, we extend to $c$-convex functions the following easy result about convex functions: if a convex function $u$ makes contact with an affine function along a line segment containing a point $x_{0}$, then either $u$ is singular along the line segment or the gradient of the affine function is an exposed point of the convex set $\partial u\left(x_{0}\right)$. Our extension is, in particular, to cost functions such that Loeper's maximum principle, Theorem 2.7 holds.

Lemma 3.3. Suppose that c satisfies (A0), (Twist), (Nondeg), and (MTW (so that Loeper's maximum principle, Theorem 2.7 (3) and its consequences, Corollary 2.8 (4) and Corollary 2.9 hold). Also let $u$ be a c-convex function on $\Omega$ and assume that $x_{0}$ is an isolated singular point of $u$. Then if $\bar{p}_{0}$ is not an extremal point of the convex set $\left[\partial_{c} u\left(x_{0}\right)\right]_{x_{0}}$ and $\bar{x}_{0}:=\exp _{x_{0}}^{c}\left(\bar{p}_{0}\right)$, the contact set

$$
S_{0}:=\left\{x \in \Omega \mid u(x)=-c\left(x, \bar{x}_{0}\right)+c\left(x_{0}, \bar{x}_{0}\right)+u\left(x_{0}\right)\right\}
$$

consists only of the single point $x_{0}$.

Proof. Fix a $\bar{p}_{0}$ that is not an extremal point of $\left[\partial_{c} u\left(x_{0}\right)\right]_{x_{0}}$. There exist $\bar{p}_{ \pm} \neq \bar{p}_{0}$ such that $\bar{p}_{ \pm} \in\left[\partial_{c} u\left(x_{0}\right)\right]_{x_{0}}$ and $\bar{p}_{0}=\frac{1}{2}\left(\bar{p}_{+}+\bar{p}_{-}\right)$; let us write $\bar{x}_{ \pm}:=\exp _{x_{0}}^{c}\left(\bar{p}_{ \pm}\right)$.

Now, suppose by contradiction that there exists some $x_{1} \in S_{0}$ with $x_{1} \neq x_{0}$. Consider the $c$-segment $x(\lambda):=\exp _{\bar{x}_{0}}^{c}\left((1-\lambda) p_{0}+\lambda p_{1}\right)$, for $\lambda \in[0,1]$ from $x_{0}$ to $x_{1}$; observe from Corollary 2.9 that $x(\lambda) \in S_{0}$ for all $\lambda \in[0,1]$. Also using that $\bar{x}_{ \pm} \in \partial_{c} u\left(x_{0}\right)$, we must have

$$
\max \left\{-c\left(x, \bar{x}_{+}\right)+c\left(x_{0}, \bar{x}_{+}\right),-c\left(x, \bar{x}_{-}\right)+c\left(x_{0}, \bar{x}_{-}\right)\right\}+u\left(x_{0}\right) \leq u(x)
$$

for all $x \in \Omega$. In particular,

$$
\begin{aligned}
-c\left(x(\lambda), \bar{x}_{ \pm}\right)+c\left(x_{0}, \bar{x}_{ \pm}\right)+u\left(x_{0}\right) & \leq u(x(\lambda)) \\
& =-c\left(x(\lambda), \bar{x}_{0}\right)+c\left(x_{0}, \bar{x}_{0}\right)+u\left(x_{0}\right)
\end{aligned}
$$

for all $\lambda \in[0,1]$. At the same time by using Theorem 2.7 (3),

$-c\left(x(\lambda), \bar{x}_{0}\right)+c\left(x_{0}, \bar{x}_{0}\right) \leq \max \left\{-c\left(x(\lambda), \bar{x}_{+}\right)+c\left(x_{0}, \bar{x}_{+}\right),-c\left(x(\lambda), \bar{x}_{-}\right)+c\left(x_{0}, \bar{x}_{-}\right)\right\}$, 
thus by combining these we must have the equality

$$
\max \left\{-c\left(x(\lambda), \bar{x}_{+}\right)+c\left(x_{0}, \bar{x}_{+}\right),-c\left(x(\lambda), \bar{x}_{-}\right)+c\left(x_{0}, \bar{x}_{-}\right)\right\}+u\left(x_{0}\right)=u(x(\lambda))
$$

for all $\lambda \in[0,1]$. Together with (9), this implies that for each $\lambda \in[0,1]$, either $\bar{x}_{+} \in \partial_{c} u(x(\lambda))$ or $\bar{x}_{-} \in \partial_{c} u(x(\lambda))$. Since $\bar{x}_{+}, \bar{x}_{-} \neq \bar{x}_{0}$ by construction, and clearly $\bar{x}_{0} \in \partial_{c} u(x(\lambda))$ for all $\lambda \in[0,1]$ this implies all points $x(\lambda)$ in the $c$-segment must be singular points, contradicting that $x_{0}$ is an isolated singular point. This proves $S_{0}=\left\{x_{0}\right\}$.

In order to prove the main theorem, we require a modified version of the estimate [12, Lemma 6.10] (this is proven in the same vein as [10, Proposition 1] for the Euclidean case of $c(x, \bar{x})=-\langle x, \bar{x}\rangle)$. By the notation $|\cdot|_{\mathcal{L}}$, we denote the volume of a set in $M, \bar{M}$ or an associated cotangent space, induced by the Riemannian metric on either $M$ or $\bar{M}$ (which will be clear from context).

Lemma 3.4. Suppose $c, u, \Omega, \bar{\Omega}, \mu$, and $\nu$ satisfy the conditions of Theorem 1.1 . Also let $m_{0}$ be a c-affine function with focus $\bar{x}_{0}$, let $S_{0}:=\left\{u \leq m_{0}\right\}$ with $S_{0} \cap \Omega^{\partial}=\emptyset$, fix two parallel planes $\Pi^{+}$and $\Pi^{-}$in $T_{\bar{x}_{0}}^{*} \bar{M}$ supporting the (convex) set $\left[S_{0}\right]_{\bar{x}_{0}}$ from opposite sides, and let $\ell_{\Pi^{ \pm}}$be the length of the longest line segment orthogonal to $\Pi^{ \pm}$that is contained in $\left[S_{0}\right]_{\bar{x}_{0}}$. Finally, suppose for some $\delta>0, x_{0} \in S_{0}^{\text {int }}$ is such that there exists $\bar{p}_{\delta} \in\left[\partial_{c} u\left(x_{0}\right) \cap(\operatorname{spt} \nu)^{\mathrm{int}}\right]_{x_{0}}$ with $d\left(\bar{p}_{\delta},\left[(\operatorname{spt} \nu)^{\partial}\right]_{x_{0}}\right) \geq \delta$. Then (writing $\left.p_{0}:=-\bar{D} c\left(x_{0}, \bar{x}_{0}\right)\right)$ there exists a constant $C>0$ depending only on $\delta, n$, $\Lambda$, diam $(\operatorname{spt} \nu)$, and $c$ such that

$$
\left(m_{0}\left(x_{0}\right)-u\left(x_{0}\right)\right)^{n} \leq \frac{C \min \left\{d\left(p_{0}, \Pi^{+}\right), d\left(p_{0}, \Pi^{-}\right)\right\}}{\ell_{\Pi^{ \pm}}}\left|S_{0}\right|_{\mathcal{L}}^{2}
$$

Proof. First, one can use (2) and follow a proof analogous to [10, Lemma 3.4] (using Remark 2.5, and replacing the Legendre transform of a function by the -transform, see [12, Section 3]), to obtain

$$
\left|\left[\partial_{c} u\left(S_{0}\right)\right]_{x_{0}} \cap[\operatorname{spt} \nu]_{x_{0}}\right|_{\mathcal{L}}=C\left|\partial_{c} u\left(S_{0}\right) \cap \operatorname{spt} \nu\right|_{\mathcal{L}} \leq \Lambda^{2} C\left|S_{0}\right|_{\mathcal{L}}
$$

where $C>0$ depends on the cost function $c$. Now let $K_{x_{0}, S_{0}}^{c}(\cdot)$ be the $c$-cone over the section $S_{0}$ with vertex $x_{0}$. Then, by using [10, Lemma 3.1], we calculate

$$
\begin{aligned}
\left|\left[\partial_{c} K_{x_{0}, S_{0}}^{c}\left(x_{0}\right)\right]_{x_{0}}\right| \mathcal{L} & \leq C(\delta, \operatorname{diam}(\operatorname{spt} \nu))\left|\left[\partial_{c} K_{x_{0}, S_{0}}^{c}\left(x_{0}\right)\right]_{x_{0}} \cap B_{\delta}\left(\bar{p}_{\delta}\right)\right|_{\mathcal{L}} \\
& \leq C(\delta, \operatorname{diam}(\operatorname{spt} \nu))\left|\left[\partial_{c} u\left(S_{0}\right)\right]_{x_{0}} \cap[\operatorname{spt} \nu]_{x_{0}}\right| \mathcal{L} \\
& \leq C\left|S_{0}\right|_{\mathcal{L}},
\end{aligned}
$$

where the final constant $C$ depends on $c, \Lambda, \delta$, and diam $(\operatorname{spt} \nu)$. Combining this with the original proof of [12, Lemma 6.10], we immediately obtain the claim.

With all of the preceeding ingredients in hand, we are ready to prove the main theorem.

Proof of Theorem 1.1. Suppose by contradiction that $u$ has an isolated singular point $x_{0} \in(\operatorname{spt} \mu)^{\text {int }}$.

We begin by a localization of $u$ around $x_{0} \cdot\left[\partial_{c} u\left(x_{0}\right)\right]_{x_{0}}$ is convex by Corollary 2.8 (4) and contains more than one point since $u$ is singular at $x_{0}$; thus there must 
exist at least one non-extremal point $\bar{p}_{0}$ of $\left[\partial_{c} u\left(x_{0}\right)\right]_{x_{0}}$. Let us define a family of sections around $x_{0}$ using $c$-affine functions with focus $\bar{x}_{0}:=\exp _{x}^{c}\left(\bar{p}_{0}\right)$, for $h>0$ let

$$
S_{h}:=\left\{x \in \Omega \mid u(x) \leq-c\left(x, \bar{x}_{0}\right)+c\left(x_{0}, \bar{x}_{0}\right)+u\left(x_{0}\right)+h\right\} .
$$

Notice that by Lemma 3.3. it holds the section is a singleton when $h=0$, i.e. $S_{0}=\left\{x_{0}\right\}$. As a result $S_{h}$ can be made sufficiently small around $x_{0}$ for small enough $h>0$. Thus by the assumption that $x_{0}$ is an isolated singularity, we may assume $h>0$ to be small enough that $S_{h} \subset(\operatorname{spt} \mu)^{\text {int }}$ and $u$ is differentiable on $S_{h} \backslash\left\{x_{0}\right\}$.

On the other hand, by Proposition 3.2 we see that the affine dimension of $\partial u\left(x_{0}\right)$ is strictly less than $n$, and in particular $\partial u\left(x_{0}\right)=\partial u\left(x_{0}\right)^{\partial}$. Hence by Proposition 3.1, the definition of Brenier solution, and closedness of $\operatorname{spt} \nu$, we see that

$$
\partial_{c} u\left(x_{0}\right)=\exp _{x_{0}}^{c}\left(\partial u\left(x_{0}\right)\right) \subset \operatorname{spt} \nu .
$$

In particular, $\bar{x}_{0} \in \operatorname{spt} \nu$. Since $u$ is differentiable on $S_{h} \backslash\left\{x_{0}\right\}$, (10) and the definition of Brenier solution imply

$$
\partial_{c} u\left(S_{h}\right) \subset \operatorname{spt} \nu
$$

Now consider the $c$-cone $K_{x_{0}, S_{h}}^{c}(x)$ over $S_{h}$ with vertex $x_{0}$ as in Lemma 2.10. From the condition spt $\nu \cap \bar{\Omega}^{\partial}=\emptyset$, it holds $\bar{x}_{0} \in \bar{\Omega}^{\text {int }}$, therefore we can apply Lemma 2.10 (5) and (6) to see

$$
-D c\left(x_{0}, \bar{x}_{0}\right) \in\left[\partial_{c} K_{x_{0}, S_{h}}^{c}\left(x_{0}\right)\right]_{x_{0}}^{\mathrm{int}} \subset\left[\partial_{c} u\left(S_{h}\right)\right]_{x_{0}} .
$$

From (11), this implies $-D c\left(x_{0}, \bar{x}_{0}\right) \in[\operatorname{spt} \nu]_{x_{0}}^{\text {int }}$.

However if this is the case, then one can follow the proof of [12, Theorem 8.3], using Lemma 3.4 above (with $\left.\delta=d\left(-D c\left(x_{0}, \bar{x}_{0}\right) \text {, [spt } \nu\right]_{x_{0}}^{\partial}\right)>0$ ) in place of [12, Theorem 6.11], to obtain that $u$ is differentiable at $x_{0}$; this contradicts that $x_{0}$ is a singular point, completing the proof.

\section{REFERENCES}

[1] Paolo Albano and Piermarco Cannarsa. Structural properties of singularities of semiconcave functions. Ann. Scuola Norm. Sup. Pisa Cl. Sci. (4), 28(4):719-740, 1999.

[2] Ralf Beyerstedt. Removable singularities of solutions to elliptic Monge-Ampère equations. Math. Z., 208(3):363-373, 1991.

[3] Ralf Beyerstedt. Isolated singularities of elliptic Monge-Ampère equations in dimensions $n \geq$ 2. Arch. Math. (Basel), 64(3):230-236, 1995.

[4] L. E. J. Brouwer. Beweis der invarianz des n-dimensionalen gebiets. Math. Ann., 71(3):305313, 1911.

[5] Luis A. Caffarelli. A localization property of viscosity solutions to the Monge-Ampère equation and their strict convexity. Ann. of Math. (2), 131(1):129-134, 1990.

[6] Luis A. Caffarelli. Some regularity properties of solutions of Monge Ampère equation. Comm. Pure Appl. Math., 44(8-9):965-969, 1991.

[7] Luis A. Caffarelli. The regularity of mappings with a convex potential. J. Amer. Math. Soc., 5(1):99-104, 1992.

[8] Piermarco Cannarsa and Yifeng Yu. Singular dynamics for semiconcave functions. J. Eur. Math. Soc. (JEMS), 11(5):999-1024, 2009.

[9] Alessio Figalli. Regularity properties of optimal maps between nonconvex domains in the plane. Comm. Partial Differential Equations, 35(3):465-479, 2010.

[10] Alessio Figalli and Young-Heon Kim. Partial regularity of Brenier solutions of the MongeAmpère equation. Discrete Contin. Dyn. Syst., 28(2):559-565, 2010. 
[11] Alessio Figalli, Young-Heon Kim, and Robert J. McCann. Continuity and injectivity of optimal maps for non-negatively cross-curved costs. arXiv:0911.3952, November 2009.

[12] Alessio Figalli, Young-Heon Kim, and Robert J. McCann. Hölder Continuity and Injectivity of Optimal Maps. Arch. Ration. Mech. Anal., 209(3):747-795, 2013.

[13] Alessio Figalli, Ludovic Rifford, and Cédric Villani. Necessary and sufficient conditions for continuity of optimal transport maps on Riemannian manifolds. Tohoku Math. J. (2), 63(4):855-876, 2011

[14] N. Guillen and J. Kitagawa. The local geometry of maps with c-convex potentials. ArXiv e-prints, December 2012. To appear in Calc. Var. Partial Differential Equations.

[15] Cristian E. Gutiérrez. The Monge-Ampère equation. Progress in Nonlinear Differential Equations and their Applications, 44. Birkhäuser Boston Inc., Boston, MA, 2001.

[16] Konrad Jörgens. Harmonische Abbildungen und die Differentialgleichung $r t-s^{2}=1$. Math. Ann., 129:330-344, 1955.

[17] Young-Heon Kim and Robert J. McCann. Continuity, curvature, and the general covariance of optimal transportation. J. Eur. Math. Soc. (JEMS), 12(4):1009-1040, 2010.

[18] Young-Heon Kim and Robert J. McCann. Towards the smoothness of optimal maps on Riemannian submersions and Riemannian products (of round spheres in particular). J. Reine Angew. Math., 664:1-27, 2012.

[19] Jiakun Liu. Hölder regularity of optimal mappings in optimal transportation. Calc. Var. Partial Differential Equations, 34(4):435-451, 2009.

[20] Jiakun Liu, Neil S. Trudinger, and Xu-Jia Wang. Interior $C^{2, \alpha}$ regularity for potential functions in optimal transportation. Comm. Partial Differential Equations, 35(1):165-184, 2010.

[21] Grégoire Loeper. On the regularity of solutions of optimal transportation problems. Acta Math., 202(2):241-283, 2009.

[22] Grégoire Loeper. Regularity of optimal maps on the sphere: the quadratic cost and the reflector antenna. Arch. Ration. Mech. Anal., 199(1):269-289, 2011.

[23] Grégoire Loeper and Cédric Villani. Regularity of optimal transport in curved geometry: the nonfocal case. Duke Math. J., 151(3):431-485, 2010.

[24] Xi-Nan Ma, Neil S. Trudinger, and Xu-Jia Wang. Regularity of potential functions of the optimal transportation problem. Arch. Ration. Mech. Anal., 177(2):151-183, 2005.

[25] Friedmar Schulz and Lihe Wang. Isolated singularities of Monge-Ampère equations. Proc. Amer. Math. Soc., 123(12):3705-3708, 1995.

[26] Neil S. Trudinger and Xu-Jia Wang. On strict convexity and continuous differentiability of potential functions in optimal transportation. Arch. Ration. Mech. Anal., 192(3):403-418, 2009.

[27] Neil S. Trudinger and Xu-Jia Wang. On the second boundary value problem for MongeAmpère type equations and optimal transportation. Ann. Sc. Norm. Super. Pisa Cl. Sci. (5), 8(1):143-174, 2009.

[28] Cédric Villani. Optimal transport: Old and new, volume 338 of Grundlehren der Mathematischen Wissenschaften [Fundamental Principles of Mathematical Sciences]. Springer-Verlag, Berlin, 2009.

[29] Yifeng Yu. Singular set of a convex potential in two dimensions. Comm. Partial Differential Equations, 32(10-12):1883-1894, 2007.

Department of Mathematics, University of British Columbia, Vancouver, V6T $1 Z 2$ CANADA

E-mail address: yhkim@math.ubc.ca

Department of Mathematics, University of Toronto, Toronto, M5S 2E4 Canada

E-mail address: kitagawa@math.toronto.edu 